\title{
The magnetic interactions in spin-glasslike $\mathrm{Ge}_{1-x-y} \mathrm{Sn}_{x} \mathrm{Mn}_{y} \mathrm{Te}$ diluted magnetic semiconductor
}

\author{
L. Kilanski $*$ R. Szymczak, and W. Dobrowolski \\ Institute of Physics, Polish Academy of Sciences, al. Lotnikow 32/46, 02-668 Warsaw, Poland \\ K. Szałowski \\ Department of Solid State Physics, University of Eódź, ul. Pomorska 149/153, 90-236 Łódź, Poland \\ V. E. Slynko and E. I. Slynko \\ Institute of Materials Science Problems, Ukrainian Academy of Sciences, 5 Wilde Street, 274001 Chernovtsy, Ukraine
}

(Dated: October 15, 2018)

\begin{abstract}
We investigated the nature of the magnetic phase transition in $\mathrm{Ge}_{1-x-y} \mathrm{Sn}_{x} \mathrm{Mn}_{y} \mathrm{Te}$ mixed crystals with chemical composition changing in the range of $0.083 \leq x \leq 0.142$ and $0.012 \leq y \leq 0.119$. The DC magnetization measurements performed in magnetic fields up to $90 \mathrm{kOe}$ and temperature range $2 \div 200 \mathrm{~K}$ showed that the magnetic ordering at temperatures below $T=50 \mathrm{~K}$ exhibits features characteristic for both spin-glass and ferromagnetic phases. The modified Sherrington - Southern model was applied to explain the observed transition temperatures. The calculations showed that the spin-glass state is preferred in the range of the experimental carrier concentrations and $\mathrm{Mn}$ contents. The value of the Mn hole exchange integral was estimated to be $J_{p d}=0.45 \pm 0.05 \mathrm{eV}$. The experimental magnetization vs temperature curves were reproduced satisfactorily using the non-interacting spin-wave theory with the exchange constant $J_{p d}$ values consistent with those calculated using modified Sherrington - Southern model. The magnetization vs magnetic field curves showed nonsaturating behavior at magnetic fields $B<90 \mathrm{kOe}$ indicating the presence of strong magnetic frustration in the system. The experimental results were reproduced theoretically with good accuracy using the molecular field approximation-based model of a disordered ferromagnet with long-range RKKY interaction.
\end{abstract}

PACS numbers: 72.80.Ga, 75.40.Cx, 75.40.Mg, 75.50.Pp

\section{INTRODUCTION}

The IV-VI group of ferromagnetic compounds e.g. $\mathrm{Ge}_{1-x} \mathrm{Mn}_{x} \mathrm{Te}$ possesses unique possibility to tune the magnetic and electrical properties of the crystals independently [1]. The magnetism of bulk (Ge,Mn)Te crystals was for the first time studied over 40 years ago by Rodot et. al [2]. The ferromagnetic ordering observed in the $\mathrm{Ge}_{1-x} \mathrm{Mn}_{x} \mathrm{Te}$ with the Curie temperatures $T_{C}$ as high as $167 \mathrm{~K} \mathrm{[3}$ ] for the crystals with the composition $x=0.5$ was attributed to the indirect long range Ruderman-Kittel-Kasuya-Yosida (RKKY) interaction. Recently, molecular beam epitaxy (MBE) grown $\mathrm{Ge}_{1-x} \mathrm{Mn}_{x}$ Te thin layers are the subject of great scientific interest $\underline{\underline{4}-\underline{9}}$. The progress in the methods of growth of this compound allowed the increase of the observed Curie temperatures to values as high as $200 \mathrm{~K}$ [6] and gives hope to obtain the room temperature ferromagnetism.

In this paper we extend our previous studies of dynamic magnetic properties of $\mathrm{Ge}_{1-x-y} \mathrm{Sn}_{x} \mathrm{Mn}_{y} \mathrm{Te}$ crystals [10, 11]. The results of the measurements of the AC susceptibilities showed the occurrence of the magnetic transitions into the spin-glasslike state at temperatures lower than $50 \mathrm{~K}$. In this paper we present the measurements of the static magnetic properties i.e. the magnetization measurements in the presence of a static magnetic field.

The observed magnetic properties are discussed in theoretical context. The Sherrington-Southern model, capturing the essentials of spin-glass physics, allows us to predict the relevant transition temperatures and to estimate the value of the exchange integral $J_{p d}$ in the studied crystals, assuming the RKKY interaction between magnetic impurities. The temperature dependence of magnetization will be modeled using the noninteracting spin wave theory, including the same RKKY interaction, which supports the estimates of $J_{p d}$. To describe the behavior of the high-field magnetization curves, we construct a molecular field approximation-based model of a disordered ferromagnet with long-range RKKY interaction, putting special emphasis on the possible magnetic inhomogenity in the system, caused by presence of antiferromagnetic superexchange interaction between nearest-neighbor magnetic ions. This model is used to explain the non-saturating behavior of magnetization.

\footnotetext{
* Electronic mail kilan@ifpan.edu.pl
} 


\section{SAMPLE PREPARATION AND BASIC CHARACTERIZATION}

For purposes of this research bulk $\mathrm{Ge}_{1-x-y} \mathrm{Sn}_{x} \mathrm{Mn}_{y} \mathrm{Te}$ crystals were prepared using modified Bridgman method. The growth method was modified according to the ideas proposed by Aust and Chalmers for the alumina crystals 12 . The radial temperature gradient was present in the growth furnace, creating $15 \mathrm{deg}$ slope of the crystallization front. It allowed the decrease of the crystal blocks in the ingot from a few down to a single one.

The chemical composition of the alloy was determined using the x-ray fluorescence method (with typical relative uncertainty of about 10\%). The as grown ingots were cut into $1 \mathrm{~mm}$ thick slices perpendicular to the growth direction. The measured chemical content changed continuously along the growth direction. For the present investigations we used the very same samples as presented in Ref. [11] i.e. the studied crystals had chemical composition changing in a range of values $0.083 \leq x \leq 0.142$ and $0.012 \leq y \leq 0.119$. The crystal slices selected for the present investigations had small inhomogeneity of the chemical composition of the order of the uncertainty of $\mathrm{x}$-ray fluorescence method i.e. less than $10 \%$ of the resultant molar fraction $x$

The crystallographic quality of $\mathrm{Ge}_{1-x-y} \mathrm{Sn}_{x} \mathrm{Mn}_{y}$ Te crystals was studied by means of the x-ray diffraction (XRD). Analysis of the XRD measurement results showed that the studied crystals were single phased. They crystallized in the [111] direction distorted $\mathrm{NaCl}$ i.e. the rhombohedral structure. The lattice parameters obtained for the studied crystals had values similar to those reported in the literature for germanium telluride bulk crystals e.g. the lattice parameter $a$ was around $5.98 \AA$ and the angle of distortion $\alpha$ was around 88.3 [deg] [13].

Basic electrical properties of $\mathrm{Ge}_{1-x-y} \mathrm{Sn}_{x} \mathrm{Mn}_{y} \mathrm{Te}$ crystals were also characterized. The measurements (using the standard DC six contact technique) of the resistivity parallel to the current direction $\rho_{x x}$ and the Hall effect (using constant magnetic field $B=14 \mathrm{kOe}$ ) for each sample at room temperature were performed. The samples had parallelepiped shape with typical diameters around $1 \times 1 \times 8 \mathrm{~mm}$. The results showed that the studied crystals were highly degenerated $p$-type semiconductors with high hole concentration $n>10^{21} \mathrm{~cm}^{-3}$ and low carrier mobilities $\mu \leq 100 \mathrm{~cm}^{2} /(\mathrm{Vs})$. For more detailed discussion of electrical properties of $\mathrm{Ge}_{1-x-y} \mathrm{Sn}_{x} \mathrm{Mn}_{y} \mathrm{Te}$ see Refs. 10 and 11.

\section{RESULTS AND DISCUSSION}

The DC magnetometry was used in the present studies of the $\mathrm{Ge}_{1-x-y} \mathrm{Sn}_{x} \mathrm{Mn}_{y}$ Te crystals. The very same sample pieces as in the case of transport characterization with electrical contacts removed were studied by means of DC magnetometry. The magnetization measurements were performed using two magnetometers. The temperature dependent magnetization in a small magnetic fields was collected using a Quantum Design SQUID MPMS XL5 magnetometer. The isothermal magnetization curves and hysteresis loops were measured using the extraction method employed by the LakeShore 7229 DC magnetometer. This method has the lower accuracy than SQUID magnetometer, but allowed us to measure at magnetic fields as high as $B=90 \mathrm{kOe}$.

\section{III.1. Low field magnetization}

The temperature dependencies of the zero-field-cooled (ZFC) and field-cooled (FC) magnetization were measured in the temperature range between 4.3 and $100 \mathrm{~K}$ using the constant magnetic field $B=50$ Oe. Typical results of the temperature dependencies of the magnetization for selected $\mathrm{Ge}_{1-x-y} \mathrm{Sn}_{x} \mathrm{Mn}_{y}$ Te crystals with constant Sn and Mn contents varied between 0.039 and 0.115 are presented in Figure 1. The differences between the FC

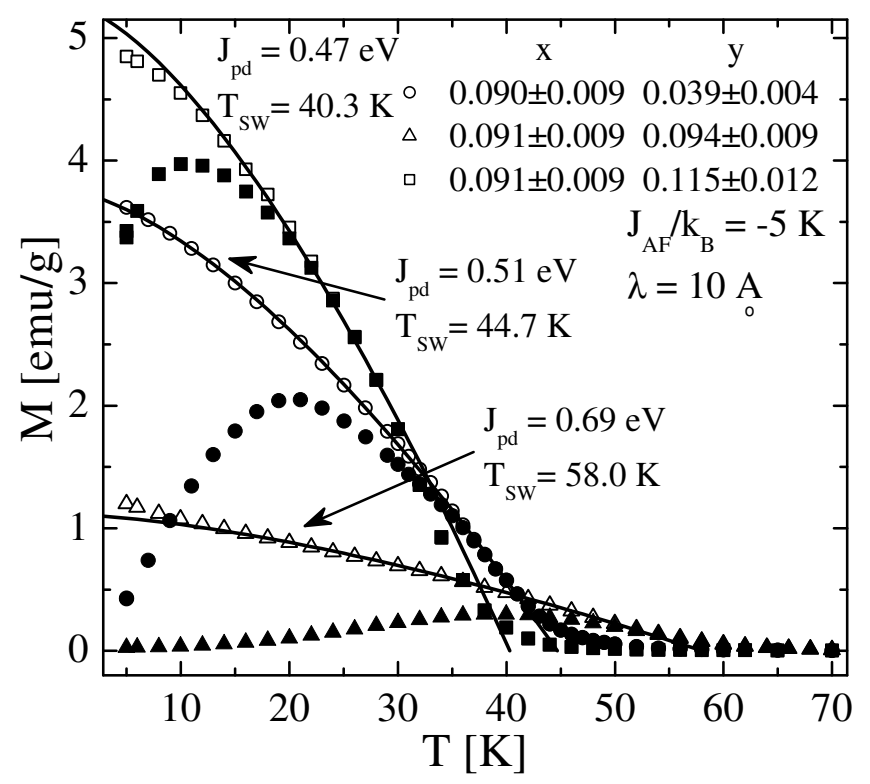

FIG. 1. Experimental (points) and theoretical (lines) magnetization as a function of temperature for samples cooled in the absence (closed symbols) and the presence (open symbols) of external magnetic field $B=50$ Oe for two selected $\mathrm{Ge}_{1-x-y} \mathrm{Sn}_{x} \mathrm{Mn}_{y} \mathrm{Te}$ samples with different chemical composition (labels).

and ZFC curves for the studied crystals were observed. Bifurcations between ZFC and FC magnetization values at low temperatures are typical features of the appearance of a spin-glass phase.

The paramagnet-spin-glass transition temperatures $T_{S G}$ were determined from the temperature dependent magnetization curves at the bifurcation points. The values obtained in this way are in good agreement with our previously determined $T_{S G}$ values from AC magnetometry results (see Ref. 11). Both the dynamic and static 
magnetic properties of $\mathrm{Ge}_{1-x-y} \mathrm{Sn}_{x} \mathrm{Mn}_{y}$ Te crystals show features similar to those observed in canonical spin-glass systems e.g. $\mathrm{CuMn}$ or AuFe diluted metallic alloys 14 .

The presented experimental FC $M(T)$ curves for weak field were theoretically reproduced with the use of the non-interacting spin wave theory. The magnon dispersion relation has been assumed in the isotropic form
$\epsilon(k)=D k^{2}$, with spin-wave stiffness coefficient $D=$ $\frac{1}{6} y S \sum_{i} J\left(R_{i j}\right) R_{i j}^{2}$, where $J\left(R_{i j}\right)$ is the RKKY exchange integral, given by a formula (1), calculated for a pair of ions in the lattice with a mutual distance of $R_{i j}$. In the present considerations it is assumed that the indirect RKKY interaction with the exchange constant $J_{R K K Y}$ has the following form $15-17$

$$
J_{R K K Y}\left(R_{i j}\right)=N_{V} \frac{m^{*} J_{p d}^{2} a_{s}^{6} k_{F}^{4}}{32 \pi^{3} \hbar^{2}} \times \frac{\sin \left(2 k_{F} R_{i j}\right)-2 k_{F} R_{i j} \cos \left(2 k_{F} R_{i j}\right)}{\left(2 k_{F} R_{i j}\right)^{4}} \exp \left(-\frac{R_{i j}}{\lambda}\right),
$$

where $m^{*}$ is the effective mass of the carriers, $J_{p d}$ is the exchange integral between conducting holes and Mn ions, $a_{0}$ is the lattice parameter, $k_{F}=\left(3 \pi^{2} n / \hbar N_{V}\right)$ is the Fermi wave vector, $N_{V}$ is the number of the valleys of the valence band, $\hbar$ is the Planck constant divided per $2 \pi$, and $R_{i j}$ is the distance between magnetic ions. For nearest-neighbor ions, the additional antiferromagnetic superexchange coupling, parameterized by $J_{A F}$, has been included. This results from averaging the magnon energies over possible orientations of wavevector $\vec{k}$ for its given length $k$ (similar to the calculation in Ref. $\underline{3}$ ).

We emphasize that using such a direction-averaged dispersion relation for our system is motivated by the presence of spatial disorder in a diluted magnetic system, which makes the influence of the exact lattice structure (involved in calculating the Fourier transform) less pronounced. Moreover, we limit our considerations to a quadratic part of the dispersion relation instead of using the full expression valid for the whole Brillouin zone. This reflects the fact that in a strongly diluted system the typical distances between the magnetic ions are noticeably larger than the lattice constant itself, so that only low-energy spin waves (of wavelength exceeding the mentioned distance) constitute well-defined excitations. The mentioned assumptions lead tho the temperature dependence of magnetization following exactly the Bloch's law in the form

$$
M(T)=M(0)\left[1-\left(T / T_{S W}\right)^{3 / 2}\right] .
$$

The characteristic temperature $T_{S W}$ can be expressed as

$$
T_{S W}=\frac{2 \pi D a^{2}}{k_{B}}\left(\frac{4 S}{\zeta(3 / 2)}\right)^{2 / 3}
$$

where $a$ is the lattice constant and $\zeta(3 / 2) \simeq 2.612$ is the appropriate Riemann zeta function. The performed calculations allowed us to satisfactorily reproduce the experimentally observed $M(T)$ curves (see Fig. 1) for the $J_{p d}$ exchange coupling constant values consistent with those obtained from the further analysis of CurieWeiss and spin-glass transition temperatures within the Sherrington-Southern model. It is worth mentioning that such a robust $T^{3 / 2}$-like behavior of $M(T)$ curves has also been noticed by [18] and [19].

The proposed theoretical approach was not able to take fully into account the magnetic frustration in the studied system, which is reflected in the shape (long tail) of the $M(T)$ curve at $T \simeq T_{S G}$.

\section{III.2. Estimation of the exchange integral $J_{p d}$}

The spin-glass state in the diluted magnetic material is well described by the model proposed by Sherrington and Southern ${ }^{20}$. The Sherrington-Southern (SS) model was found to be the most appropriate approach for the description of systems with a large number of neighboring paramagnetic ions. Moreover, the modified SS model was applied to compounds belonging to the group of IVVI semimagnetic semiconductors i.e. $\mathrm{Sn}_{1-x} \mathrm{Mn}_{x} \mathrm{Te}$ and $\mathrm{Pb}_{1-x-y} \mathrm{Sn}_{x} \mathrm{Mn}_{y} \mathrm{Te}$ [21]. In the present work we adapted the modified SS model [21] to quantify the $J_{p d}$ exchange interaction in the studied $\mathrm{Ge}_{1-x-y} \mathrm{Sn}_{x} \mathrm{Mn}_{y} \mathrm{Te}$ mixed crystals. Sherrington and Southern proposed the Gaussian distribution of the interaction strength in Heisenberg Hamiltonian, with mean value $J_{0}$ and width $\Delta J$. The model allows the calculations of both paramagnetic Curie-Weiss $\Theta$ and paramagnet-spin-glass transition $T_{S G}$ temperatures given by Eqs. 4 and 5

$$
\begin{aligned}
\Theta & =\frac{2 S(S+1) x}{3 k_{B}} J_{0}, \\
T_{S G} & =\frac{2 \Delta J}{3 k_{B}}\left[S^{2}(S+1)^{2}+S(S+1) / 2\right]^{1 / 2},
\end{aligned}
$$

where $k_{B}$ is the Boltzmann constant. In the above equations the RKKY indirect interactions has the exchange constant $J_{R K K Y}$ is expressed using the equation 1. We assumed that the band structure of the studied $\mathrm{Ge}_{1-x-y} \mathrm{Sn}_{x} \mathrm{Mn}_{y}$ Te crystals is similar to that of GeTe. GeTe has got a nearly spherical valence band with maximum at the $L$-point of the Brillouin zone 22 the effective mass of the carriers in the four-fold degenerated $L$-band equal to $1.2 \cdot m_{e}$ [23], where $m_{e}$ is the free electron mass, as in the case of the GeTe crystals. The SS model assumes the finite range of the interactions by introducing the exponential damping factor $\lambda$. In our calculations we 


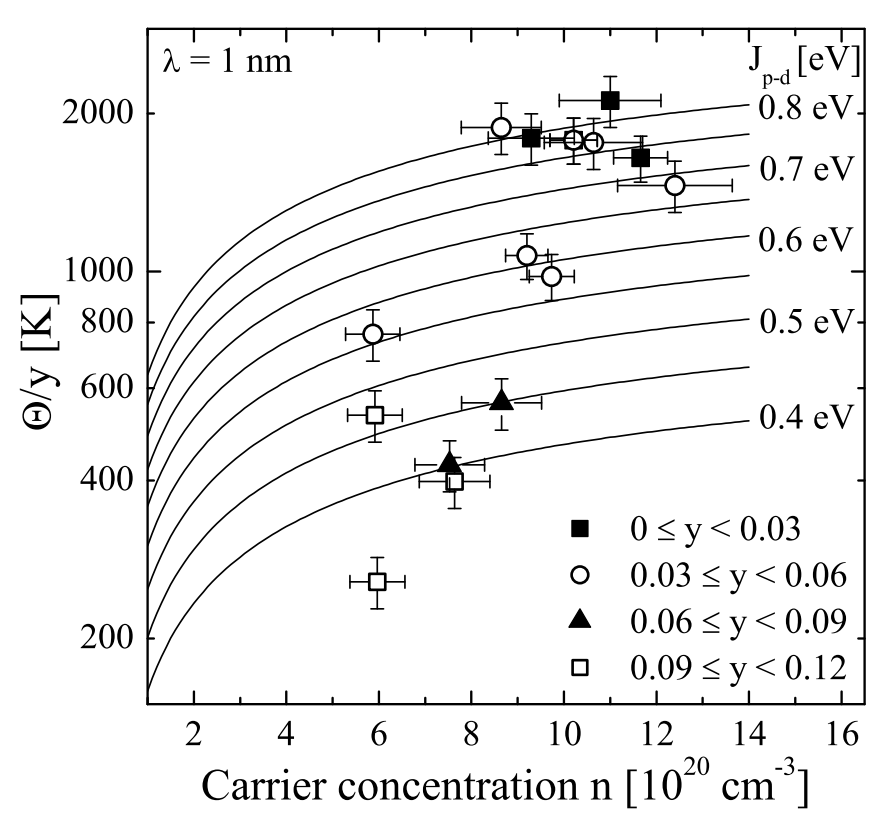

FIG. 2. The Curie-Weiss temperature normalized to the Mn molar fraction $\mathrm{y}$ as a function of the carrier concentration $n$ calculated theoretically (lines) using values of the exchange integral $J_{p d}$ varying between $0.4 \mathrm{eV}$ to $0.8 \mathrm{eV}$ with step $0.05 \mathrm{eV}$, and the experimental values (points) obtained for the studied $\mathrm{Ge}_{1-x-y} \mathrm{Sn}_{x} \mathrm{Mn}_{y}$ Te crystals grouped with respect to the amount of $\mathrm{Mn}$ ions in the alloy $y$ (see legend).

assumed $\lambda$ to be equal to $1 \mathrm{~nm}$ what is a reasonable value for the semiconductor with relatively low carrier mobility $\mu<100 \mathrm{~cm}^{2} /(\mathrm{Vs})$ (see Refs. 10 and 11). The lattice parameters for the pure GeTe crystals were used in our calculations.

The results of calculation of the $\Theta(n)$ dependencies performed for values of the Mn-hole exchange integral $J_{p d}$ varying between 0.4 and $0.8 \mathrm{eV}$ are presented in Fig. 2 , Most of the experimental points lie between the $\Theta(n)$ curves calculated for $J_{p d}$ varying between the two values $0.4 \div 0.8 \mathrm{eV}$. Because of the large scatter of the experimental values we found it reasonable to focus on the spin-glass transition temperature $T_{S G}$ estimations which as will be seen give more accurate results.

The $T_{S G}$ dependence on the Mn content for a few values of the hole concentrations is presented in Fig. 3. The experimentally determined values of $T_{S G}(y)$ are well described by the theoretical curves with the values of the exchange constant $J_{p d}$ changing between 0.4 and $0.5 \mathrm{eV}$. It must be noted, that for the samples for which the $T_{S G}$ values are in the vicinity of $J_{p d}=0.5 \mathrm{eV}$, the coercive fields were the highest in the entire series of $\mathrm{Ge}_{1-x-y} \mathrm{Sn}_{x} \mathrm{Mn}_{y} \mathrm{Te}$ crystals. It may be interpreted that the estimated higher value of the exchange constant for these crystals is not an artifact but reflects their magnetic properties.

The estimated values of $J_{p d}$ for the studied $\mathrm{Ge}_{1-x-y} \mathrm{Sn}_{x} \mathrm{Mn}_{y} \mathrm{Te}$ crystals are within the range between reported in literature for $\mathrm{Sn}_{1-x} \mathrm{Mn}_{x}$ Te bulk crys-

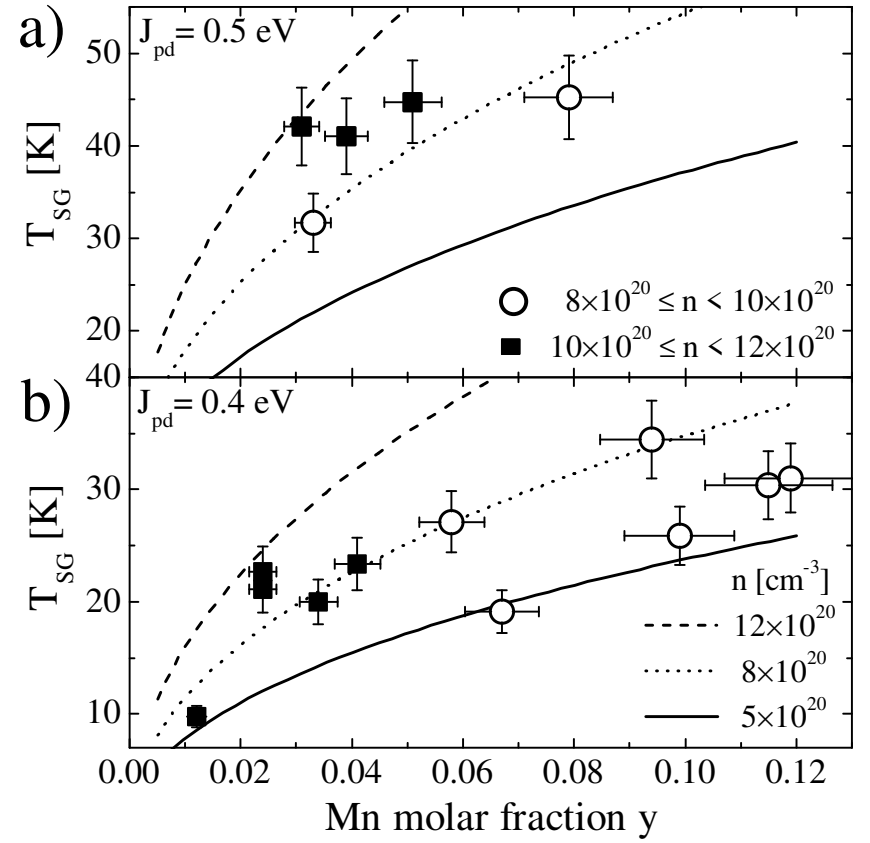

FIG. 3. The spin glass transition temperatures $T_{S G}$ as a function of the Mn molar fraction $y$ calculated (lines) for two different values of the exchange integral $J_{p d}$ and different values of carrier concentration $n$ (see legend), and the experimentally determined values of $T_{S G}$ for $\mathrm{Ge}_{1-x-y} \mathrm{Sn}_{x} \mathrm{Mn}_{y} \mathrm{Te}$ crystals with different chemical content $y$. The points were grouped with respect to the different carrier concentrations (see legend).

tals 24] $\left(J_{p d} \approx 0.1 \mathrm{eV}\right.$ for $\left.x \leq 0.1\right)$ and $\mathrm{Ge}_{1-x} \mathrm{Mn}_{x} \mathrm{Te}$ $\left(J_{p d} \approx 0.8 \div 0.9 \mathrm{eV}\right.$ for $\left.x>0.1\right)$ bulk crystals $[18,[3]$, and $J_{p d} \approx 0.7 \mathrm{eV}$ for $x=0.07$ as obtained in $\mathrm{Ge}_{1-x} \mathrm{Mn}_{x}$ Te epitaxial layers 25].

\section{III.3. High field magnetization}

The magnetic properties of $\mathrm{Ge}_{1-x-y} \mathrm{Sn}_{x} \mathrm{Mn}_{y}$ Te crystals is the presence of high magnetic field for $B \leq 90 \mathrm{kOe}$ were studied at temperatures between $4.3 \leq T \leq 80 \mathrm{~K}$. At magnetic fields $B \leq 600$ Oe an irreversible behavior with clear hysteresis is observed. Typical results of hysteresis curves recorded for a $\mathrm{Ge}_{0.871} \mathrm{Sn}_{0.090} \mathrm{Mn}_{0.039} \mathrm{Te}$ sample at a few

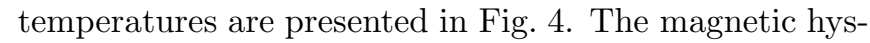
teresis is not a typical feature in canonical spin-glasses such as CuMn [14] but was observed for many spin-glass systems such as AuFe with $8 \%$ at. Fe [26] or NiMn with $21 \%$ at. Mn 27.

The analysis of hysteresis loops showed that there exists a correlation between the Mn content $y$, the values of spontaneous magnetization $M_{R}$, and the coercive field $H_{C}$ (see Fig. 4b). When $y$ changes between 0.012 and 0.039 the values (measured at $T \approx 4.5 \mathrm{~K}$ ) of $M_{R}$ increase from $0.45 \mathrm{emu} / \mathrm{g}$ to $2.5 \mathrm{emu} / \mathrm{g}$ and $H_{C}$ values are increasing from 80 Oe to 430 Oe. In the case of the group of crystals with $y>0.039$ the inverse dependencies were 

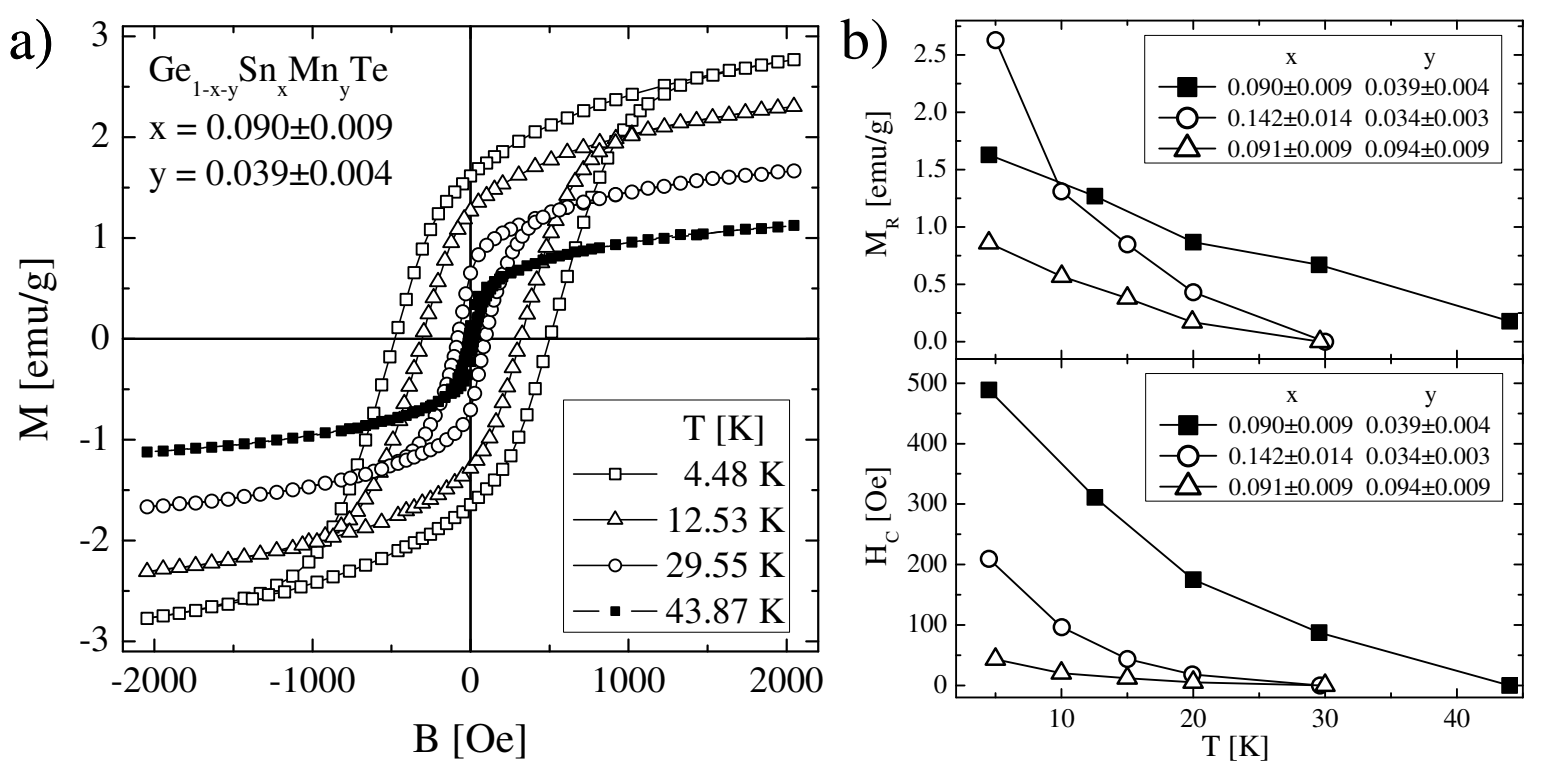

FIG. 4. a) Hysteresis loops measured at selected constant temperatures (see legend) for selected $\mathrm{Ge}_{1-x-y} \mathrm{Sn}_{x} \mathrm{Mn}_{y} \mathrm{Te}$ sample (chemical composition shown in legend) and b) spontaneous magnetization $M_{R}$ and coercive field $H_{C}$ as a function of temperature for a few selected $\mathrm{Ge}_{1-x-y} \mathrm{Sn}_{x} \mathrm{Mn}_{y}$ Te samples with different chemical composition (see legend).

observed, namely, the reduction of both $M_{R}$ and $H_{C}$ with increasing $y$. In the case of the group of crystals with Mn content $\approx 0.035$ and Sn content $x$ changing between $x=0.090$ and 0.145 , the changes of the $H_{C}$ and $M_{R}$ can be attributed to the increase of the carrier concentration. The coercive field $H_{C}$ becomes smaller with increasing amount of tin ions changing from 430 Oe to 210 Oe for crystals with $x=0.090$ and 0.145 . In turn, the value of $M_{R}$ showed the opposite trend rising from 1.6 to $2.6 \mathrm{emu} / \mathrm{g}$.

The observed parameters of the magnetic hysteresis loop and their changes with the chemical composition of the alloy can be associated with the modification of the domain structure of the material. Furthermore, together with the increasing Mn content the probability for antiferromagnetic pairing of $\mathrm{Mn}$ ions increases.

The $H_{C}$ values observed in our studies are smaller than reported in the literature for bulk $\mathrm{Ge}_{1-x} \mathrm{Mn}_{x}$ Te crystals [2] $\left(H_{C}<1200\right.$ Oe for $\left.x=0.048\right)$, but are close to those reported for thin $\mathrm{Ge}_{1-x} \mathrm{Mn}_{x}$ Te epitaxial layers 25] $\left(H_{C}<700\right.$ Oe for $\left.x<0.6\right)$.

The typical results for the $\mathrm{Ge}_{0.824} \mathrm{Sn}_{0.142} \mathrm{Mn}_{0.034} \mathrm{Te}$ crystal obtained in the range of magnetic fields up to $90 \mathrm{kOe}$ and temperatures lower than $80 \mathrm{~K}$ are presented in Fig. 5. The $M(B)$ curves do not saturate and are nearly linear in the range of magnetic fields $B \geq 30 \mathrm{kOe}$. The lack of saturation of $M$ reflects the fact that the studied alloy is not a ferromagnet but a spin glass at the temperatures below $50 \mathrm{~K}$

In order to model the field dependence of magnetization, we performed calculations within the molecularfield approximation, constructing the model described

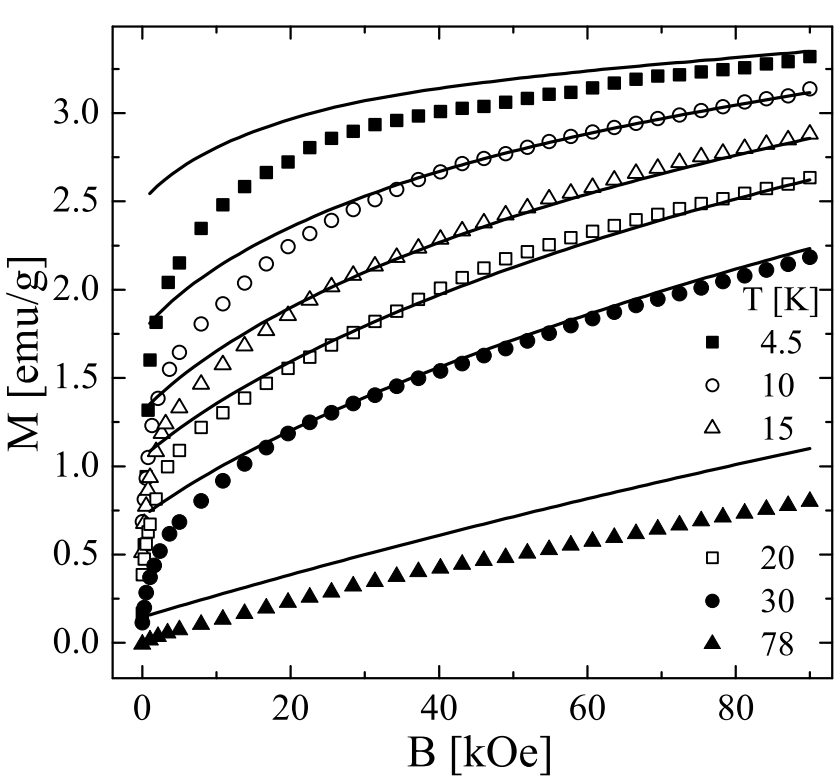

FIG. 5. Isothermal magnetization curves $M(B)$ measured (points) and reproduced using the mean field model described in the Appendix (lines) for the parameters $J_{p d}=0.75 \mathrm{eV}$, $y=0.034, p=0.71, \lambda=15 \AA, J_{A F} / k_{\mathrm{B}}=-20 \mathrm{~K}$ at different temperatures (see legend) for selected $\mathrm{Ge}_{1-x-y} \mathrm{Sn}_{x} \mathrm{Mn}_{y} \mathrm{Te}$ sample with $x=0.142 \pm 0.014$ and $y=0.034 \pm 0.003$.

in the Appendix. The numerical calculations were performed for the exchange constant $J_{p d}$, the Mn amount $y$, the fraction of isolated magnetic ions $p$, the interaction decay distance $\lambda$, and the energy of the antiferromag- 


\section{SUMMARY AND CONCLUSIONS}

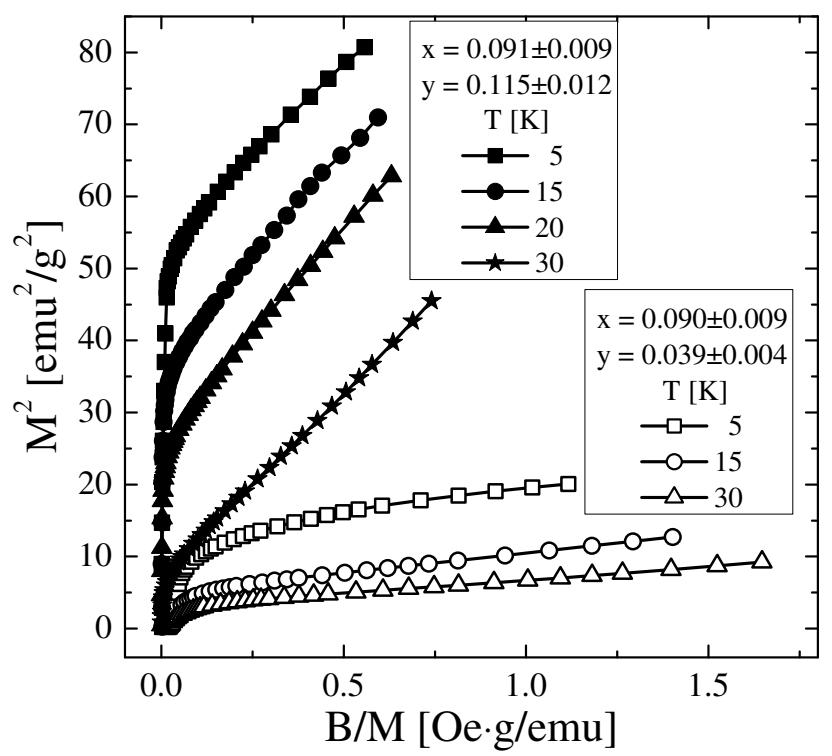

FIG. 6. The Arrot plot for two selected $\mathrm{Ge}_{1-x-y} \mathrm{Sn}_{x} \mathrm{Mn}_{y} \mathrm{Te}$ crystals with different chemical composition (see legend).

netic interactions in the system $J_{A F}$ specified and for $2 \cdot 10^{4}$ realizations of disorder, summing the interactions between the magnetic ions up to the distance of $100 \AA$. The method allowed to satisfactorily reproduce the highfield behavior of $M(H)$ curves, i.e. the linear increase of magnetization with the field. It must be emphasized that the method adopted is based on the molecular-field approximation, so that it cannot be expected to account for the collective excitations in the system and to yield the precise temperature dependence of magnetization. It is obvious that the low-field magnetic susceptibility is underestimated by the present model, which might be attributed to neglect of correlations.

In Fig. 6 the isothermal magnetization curves obtained for the studied crystals are presented in the form of Arrot plots. With the increase of the $\mathrm{Mn}$ content in the samples the experimental $M^{2}(B / M)$ curves change their character. Such a behavior is usually attributed to the change of the strength of the ferromagnetic interactions in the system. In the case of the $\mathrm{Ge}_{1-x-y} \mathrm{Sn}_{x} \mathrm{Mn}_{y} \mathrm{Te}$ samples with Mn content $y \leq 0.039$ the observed behavior is similar to the one observed in many canonical spin-glass systems with predominance of the magnetic interactions with the exchange constant $J>0$ [28]. It is well known that in case of a spin-glass systems the $M^{2}(B / M)$ curves on the Arrot plot lies on the bottom side of the plot what reflects the absence of spontaneous magnetization in the system. With the increase of the amount of $\mathrm{Mn}$ above $y=0.039$ the $M^{2}(B / M)$ curves on the Arrot plot shifts towards the top-left side of the plot. This is a signature that the ferromagnetic interaction dominates in the studied samples [28]. Nevertheless, the system remains in the frustrated spin-glass state (see Fig. [6).
We presented detailed studies of the magnetic properties of the $\mathrm{Ge}_{1-x-y} \mathrm{Sn}_{x} \mathrm{Mn}_{y} \mathrm{Te}$ crystals with different amount of both magnetic and nonmagnetic constituents i.e. $\mathrm{Mn}$ and $\mathrm{Sn}$ ions.

The DC magnetometry measurements showed that the studied spin-glass system shows some features characteristic of a ferromagnetic material. Hysteresis loops with large coercive fields (maximum of $500 \mathrm{Oe}$ ) and spontaneous magnetization were observed indicating that the studied system possessed features characteristic of a ferromagnetic material. The high field magnetization curves showed nonsaturating behavior at magnetic fields as high as $90 \mathrm{kOe}$ which was interpreted as a direct proof of a strong magnetic frustration in this system. The observed bifurcations of the FC/ZFC magnetization $M(T)$ dependencies as well as the non-saturating $M(B)$ curves are the features characteristic for spin-glasses. The experimental $M(T)$ curves were reproduced theoretically using the non-interacting spin wave theory. The $M(B)$ curves were fitted theoretically using the molecular field approximation. Theoretical calculations reproduced well the experimental results especially in the range of high magnetic fields. The linear $M(B)$ dependence was reproduced by including magnetic disorder in the theoretical model and separate treatment of the antiferromagnetically-coupled pairs of magnetic ions. The proposed model gives satisfactory agreement with the experimental curves only in the case of the presence of the antiferromagnetic nearestneighbor interactions in the system. This is an evidence that strong magnetic disorder is present in the studied system.

The calculations based on a modified SherringtonSouthern model showed that the spin glass state is the preferred magnetic state for the experimentally determined hole concentrations as well as Mn molar fractions. The calculations allowed us to reproduce with good accuracy the spin glass transition temperatures for all the studied crystals and to estimate the Mn-hole exchange integral value to be around $J_{p d}=0.45 \pm 0.05 \mathrm{eV}$ for the studied $\mathrm{Ge}_{1-x-y} \mathrm{Sn}_{x} \mathrm{Mn}_{y} \mathrm{Te}$ mixed crystals.

\section{ACKNOWLEDGMENTS}

The authors would like to thank Prof. T. Balcerzak for helpful discussions.

This work was supported by the Ministry of Science and Higher Education of Poland from a funds for science in years 2009-2010 as a scientific project no. N N202 236537. 


\section{Appendix: Theoretical model including antiferromagnetic interactions}

The hamiltonian of the system of interacting magnetic ions distributed over the lattice sites is:

$$
\mathcal{H}=-\frac{1}{2} \sum_{i, j} J\left(R_{i j}\right) \xi_{i} \xi_{j} \mathbf{S}_{i} \mathbf{S}_{j}-g_{S} \mu_{B} B \sum_{i} \xi_{i} \mathbf{S}_{i}^{z},
$$

where the operators $\mathbf{S}_{i}$ describe the spins $S=5 / 2$ distributed over $N$ fcc lattice sites (the rhombohedral structure of $\mathrm{Ge}_{1-x-y} \mathrm{Sn}_{x} \mathrm{Mn}_{y}$ is very close to the fcc structure). The exchange integral is given by Eq. (11). The disorder in the site-diluted magnetic system is introduced by occupation number operators $\xi_{i}$ taking the values of 0,1 for each lattice site $i$. The set of $\xi_{i}$ values describes completely the given realization of the disorder in the system.

Because of presence of the disorder, the lattice sites occupied by magnetic ions are inequivalent in the thermodynamic meaning. The most pronounced source of inhomogenity is due to additional superexchange coupling occurring between the magnetic ions in nearestneighbor position. In order to account for this, the system of magnetic impurities located on lattice sites (with atomic concentration equal to $y$ ) was subdivided into two (interacting) subsystems. For low values of $y$ the significant number of impurity ions can be expected to lack the other magnetic impurities in the nearest-neighbor position. Therefore, such magnetic ions do not couple antiferromagnetically to other magnetic ions via the superexchange mechanism. Let us denote the total number of such ions (called further 'isolated magnetic ions') by $N p y$. For purely random, uncorrelated occupation of fcc lattice sites by impurities, the probability that a selected magnetic ion is isolated equals to $p=(1-y)^{12}$ (what yields the value of 0.66 for $y=0.034)$. However, if the distribution of magnetic impurities is not completely random, $p$ deviates from the above mentioned value $\left(p<(1-y)^{12}\right.$ for ion clustering tendency, $p>(1-y)^{12}$ for the opposite tendency).

We assume approximately that the remaining magnetic ions form isolated nearest-neighbor pairs (i.e. pairs of ions that do not possess further magnetic nearest neighbors). Within each pair, both ions are coupled via the superexchange mechanism, characterized by the exchange integral $J_{A F}<0$. We neglect the possibility of formation of magnetic clusters containing more than two superexchange-coupled magnetic ions, which is justified for small $y$. According to this assumption, the number of isolated pairs is $\frac{1}{2} y(1-p)$. Let us emphasize that due to the neglect of larger magnetic clusters, the value of parameter $p$ determined from the best-fit to the experimental data may differ from the actual fraction of isolated magnetic ions in the real system.

To construct the thermodynamic description of the model, we apply a molecular-field approximation, assuming that the total state of the system takes the form of a tensor product of the appropriate single-site density matrices. The quantum state of each site occupied by an isolated magnetic ion is described by a density matrix of the form

$$
\boldsymbol{\rho}_{i, 0}=\exp \left[\left(\lambda_{0}+g_{S} \mu_{B} B\right) \mathbf{S}_{i}^{z} / k_{\mathrm{B}} T\right] / Z_{0},
$$

with $Z_{0}=\sum_{s=-S}^{S} \exp \left[\left(\lambda_{0}+g_{S} \mu_{B} B\right) s / k_{\mathrm{B}} T\right]$. The thermodynamic average of the spin value of such an ion equals to

$$
\left\langle\mathbf{S}_{i}^{z}\right\rangle=m_{0}=S \mathcal{B}_{S}\left(\frac{\lambda_{0}+g_{S} \mu_{B} B}{k_{\mathrm{B}} T}\right),
$$

$S \mathcal{B}_{S}(x)$ being the Brillouin function for spin $S$.

Let us further denote one of the (inequivalent) ions belonging to an isolated nearest-neighbor pair by + , while the other one by - . The corresponding density matrices are assumed in the form:

$$
\boldsymbol{\rho}_{i, \pm}=\exp \left[\left(\lambda_{ \pm}+g_{S} \mu_{B} B\right) \mathbf{S}_{i}^{z} / k_{\mathrm{B}} T\right] / Z_{ \pm}
$$

with $Z_{ \pm}=\sum_{s=-S}^{S} \exp \left[\left(\lambda_{ \pm}+g_{S} \mu_{B} B\right) s / k_{\mathrm{B}} T\right]$. The thermodynamic average of spin value of each of the ions is

$$
\left\langle\mathbf{S}_{i}^{z}\right\rangle=m_{ \pm}=S \mathcal{B}_{S}\left(\frac{\lambda_{ \pm}+g_{S} \mu_{B} B}{k_{\mathrm{B}} T}\right)
$$

The parameters $\lambda_{0}, \lambda_{+}$and $\lambda_{-}$are the variational parameters of the molecular field.

For such a model, the total entropy is a sum of contributions originating from each single isolated magnetic ion $\left(-k_{\mathrm{B}} \operatorname{Tr}\left[\boldsymbol{\rho}_{i, 0} \ln \boldsymbol{\rho}_{i, 0}\right]\right)$ and from each isolated magnetic pair $\left(-k_{\mathrm{B}} \operatorname{Tr}\left[\boldsymbol{\rho}_{i,+} \ln \boldsymbol{\rho}_{i,+}\right]-k_{\mathrm{B}} \operatorname{Tr}\left[\boldsymbol{\rho}_{i,-} \ln \boldsymbol{\rho}_{i,-}\right]\right)$. The total entropy of the system is:

$$
\begin{array}{r}
\mathcal{S}=k_{\mathrm{B}} \sum_{i} \xi_{i}\left[p \ln Z_{i, 0}+\frac{1-p}{2}\left(\ln Z_{i,+}+\ln Z_{i,-}\right)+\right. \\
\left.-p \frac{\lambda_{0}+g_{S} \mu_{B} B}{k_{\mathrm{B}} T} m_{0}-\frac{1-p}{2}\left(\frac{\lambda_{+}+g_{S} \mu_{B} B}{k_{\mathrm{B}} T} m_{+}+\frac{\lambda_{-}+g_{S} \mu_{B} B}{k_{\mathrm{B}} T} m_{-}\right)\right] .
\end{array}
$$

The enthalpy of the model is a thermodynamic average of the Hamiltonian, containing the terms describing the interaction between the isolated ions, between the isolated magnetic pairs as well as the interaction between 


$$
\begin{aligned}
\langle\mathcal{H}\rangle & =-\sum_{i} \xi_{i}\left\{\frac{1}{2} p^{2} m_{0}^{2} \sum_{j} \xi_{j} J\left(R_{i j}\right)+p m_{0} g_{S} \mu_{B} B+\frac{1}{2}(1-p) J_{A F} m_{+} m_{-}+\right. \\
& +\frac{1-p}{2} g_{S} \mu_{B} B\left(m_{+}+m_{-}\right)+p \frac{1-p}{2} m_{0}\left(m_{+}+m_{-}\right) \sum_{j} \xi_{j} J\left(R_{i j}\right)+ \\
& \left.+\frac{1}{4}(1-p)^{2} \sum_{j} \xi_{j} J\left(R_{i j}\right)\left(m_{+}+m_{-}\right)^{2}\right\} .
\end{aligned}
$$

Let us note that in the formula above, as well as in the rest of the Appendix, the nearest-neighbors of the lattice site $i$ will be excluded from summation over $j$.

The total Gibbs free energy of the system is obtained from the expression $G=\langle\mathcal{H}\rangle-\mathcal{S} T$. The variational minimization of the Gibbs energy with respect to molecular field parameters yields the set of coupled self-consistent equation of the form:

$\sum_{i} \xi_{i}\left\{\lambda_{0}-\left[p m_{0}+\frac{1-p}{2}\left(m_{+}+m_{-}\right)\right] \sum_{j} \xi_{j} J\left(R_{i j}\right)\right\}=0$

$\sum_{i} \xi_{i}\left\{\lambda_{+}-J_{A F} m_{-}-p m_{0} \sum_{j} \xi_{j} J\left(R_{i j}\right)\right\}=0$

$\sum_{i} \xi_{i}\left\{\lambda_{-}-J_{A F} m_{+}-p m_{0} \sum_{j} \xi_{j} J\left(R_{i j}\right)\right\}=0$.

Then we are looking for the solution when all the terms under the sum over $i$ vanish simultaneously, i.e. we obtain the following set of equations :

$$
\lambda_{0}=\left[p m_{0}+\frac{1-p}{2}\left(m_{+}+m_{-}\right)\right] \sum_{j} \xi_{j} J\left(R_{j}\right)
$$

$$
\begin{aligned}
& \lambda_{+}=J_{A F} m_{-}+p m_{0} \sum_{j} \xi_{j} J\left(R_{j}\right) \\
& \lambda_{-}=J_{A F} m_{+}+p m_{0} \sum_{j} \xi_{j} J\left(R_{j}\right),
\end{aligned}
$$

which need to be solved together with the conditions (A.3) and (A.5). The solution allows us to calculate the total magnetization in the system for a given realization of disorder as:

$$
M=n_{m} g_{S} \mu_{B}\left[p m_{0}+\frac{1-p}{2}\left(m_{+}+m_{-}\right)\right] .
$$

Finally, to obtain the disorder-averaged site magnetization, we average the values obtained from the Eq. A.10 for a sufficiently large number of realizations of disorder. Each realization of disorder is simulated numerically, by allowing the parameters $\xi_{j}$ to take the values of 0,1 according to the probability distribution $p\left(\xi_{j}\right)=y \delta\left(\xi_{j}-1\right)+(1-y) \delta\left(\xi_{j}\right)$, with the total concentration of magnetic impurities equal to $y$.
1 Y. Fukuma, H. Asada, M. Arifuku, and T. Koyanagi, Appl. Phys. Lett. 80, 1013 (2002).

2 M. Rodot, J. Lewis, H. Rodot, G. Villers, J. Cohen, and P. Mollard, J. Phys. Soc. Japan Suppl. 21, 627 (1966).

3 R. W. Cochrane, M. Plischke, and J. O. Töin-Olsen, Phys. Rev. B 9, 3013 (1974).

4 Y. Fukuma, H. Sato, K. Fujimoto, K. Tsuji, A. Kimura, M. Taniguchi, S. Senba, A. Tanaka, H. Asada, and T. Koyanagi, J. Appl. Phys. 99, 08D510 (2006).

5 W. Q. Chen, K. L. Teo, M. B. A. Jalil, and T. Liew, J. Appl. Phys. 99, 08D515 (2006).

${ }^{6}$ R. T. Lechner, R. Kirchschlager, G. Springholz, T. Schwarzl, and G. Bauer, in Narrow Gap Semiconductors 13 url: http://www.ati.surrey.ac.uk/NGS13/presentations/MO3_4.kpdf (2007).
7 W. Knoff, V. Domukhovski, K. Dybko, P. Dziawa, M. Gorska, R. Jakiela, E. Lusakowska, A. Reszka, B. Taliashvili, T. Story, J. R. Anderson, and C. R. Rotundu, Acta Phys. Pol. A 114, 1159 (2008).

8 S. T. Lim, J. F. Bi, K. L. Teo, Y. P. Feng, T. Liew, and T. C. Chong, Appl. Phys. Lett. 95, 072510 (2009).

9 B. J. Kowalski, M. A. Pietrzyk, W. Knoff, A. Łusakowski, J. Sadowski, J. Adell, and T. Story, Physics Procedia 3, 1357 (2010).

10 L. Kilanski, M. Arciszewska, V. Domukhovsky, W. Dobrowolski, V. E. Slynko, and E. I. Slynko, Acta Phys. Pol. A 114, 1145 (2008).

11 L. Kilanski, M. Arciszewska, V. Domukhovsky, W. Dobrowolski, V. E. Slynko, and E. I. Slynko, J. Appl. Phys. 105, 103901 (2009).

12 K. T. Aust and B. Chalmers, Can. J. Phys. 36, 977 (1958). 
13 R. R. Galazka, J. Kossut, and T. Story, "Landoltbörnstein, new series, group iii/41," (Berlin, Heidelberg, Springer-Verlag, 1999) Chap. Semiconductors.

14 J. A. Mydosh, Spin Glasses: An Experimental Introduction (Taylor and Francis, London, 1994).

15 M. A. Ruderman and C. Kittel, Phys. Rev. 96, 99 (1954).

16 T. Kasuya, Progr. Theor. Phys. 16, 45 (1956).

17 K. Yoshida, Phys. Rev. 106, 893 (1957).

18 R. W. Cochrane, F. T. Hedgcock, and J. O. Ström-Olsen, Phys. Rev. B 8, 4262 (1973).

19 Y. Fukuma, H. Asada, S. Miyawaki, T. Koyanagi, S. Senba, K. Goto, and H. Sato, Appl. Phys. Lett. 93, 252502 (2008).

20 D. Sherrington and B. W. Southern, J. Phys. F: Met. Phys. 5, L49 (1975).

${ }^{21}$ P. J. T. Eggenkamp, H. J. M. Swagten, T. Story, V. I. Litvinov, C. H. W. Swüste, and W. J. M. de Jonge, Phys.
Rev. B 51, 15250 (1995).

22 J. E. Lewis, Phys. Stat. Sol. B 35, 737 (1969).

23 J. E. Lewis, Phys. Stat. Sol. B 59, 367 (1973).

24 P. J. T. Eggenkamp, C. W. H. M. Vennix, T. Story, H. J. M. Swagten, C. H. W. Swüste, and W. J. M. de Jonge, J. Appl. Phys. 75, 5728 (1994).

25 Y. Fukuma, H. Asada, N. Nishimura, and T. Koyanagi, J. Appl. Phys. 93, 4034 (2003).

26 J. J. Prejean, M. J. Joliclerc, and P. Monod, J. Phys. (Paris) 41, 427 (1980).

27 S. Senoussi, J. Phys. 45, 315 (1984).

28 J. G. Sereni, E. Beaurepaire, and J. P. Kappler, Phys. Rev. B 48, 3747 (1993). 